\title{
EXPLORING THE EFFECTS OF EXPERIENCE ON WIKI ANXIETY AND WIKI USABILITY: AN ONLINE STUDY
}

\author{
Benjamin R. Cowan \\ Institute for Digital Communications \\ University of Edinburgh \\ King's Buildings, EH9 3JL \\ +441316508231 \\ b.cowan@ed.ac.uk
}

\author{
Lorenzo Vigentini \\ Psychology Department \\ University of Edinburgh \\ 7 George Square, EH8 9JZ \\ +44131650 3446 \\ I.vigentini@ed.ac.uk
}

\author{
Mervyn A. Jack \\ Institute for Digital Communications \\ University of Edinburgh \\ King's Buildings, EH9 3JL \\ +441316502783 \\ m.a.jack@ed.ac.uk
}

\begin{abstract}
Information Technology is now pervasive in Higher Education institutions and developments in IT are changing the technological landscape at Universities. A recent phenomenon shaping such changes is the use of Web 2.0 tools in a pedagogical context. These tools are often included into a University's IT mix without full appreciation of the possible negative emotions student users may have towards these tools. It is generally assumed that experience with the IT system will be enough to reduce any anxious feelings which may manifest in users about such systems. This study firstly aims to observe the relationship that such emotion may have on usability evaluation of a wiki system. It also aims to investigate the effect of experience on students' negative affective reactions towards a wiki tool. Second year undergraduate psychology students ( $\mathrm{N}=92)$ who were using a wiki to collaborate on course projects completed questionnaires measuring usability evaluation and anxiety towards the wiki both 2 weeks (Time 1) and 12 weeks (Time 2) into their usage of the system. The research found that wiki anxiety was negatively correlated to participants' usability evaluations of the wiki at both time 1 and time 2. Further experience with the system had little effect on users' negative emotions towards the wiki. Users also showed little change in their usability rating of the system with more exposure to the wiki. However any change in wiki anxiety over the study was negatively correlated with a change in usability evaluation. Possible interpretations of the relationship between wiki anxiety, wiki usability and possible effects of the type and quality of user experience on wiki anxiety are discussed.
\end{abstract}

\section{Categories and Subject Descriptors}

\section{General Terms}

Experimentation, Human Factors.

\section{Keywords}

Wiki, Wiki anxiety, usability, experience

(C) The Author 2009.

Published by the British Computer Society

\section{INTRODUCTION}

Information Technology (IT) has become an integral aspect of educational practice. In higher education (HE), IT provides core resources, ranging from the dissemination of course material (e.g. lecture notes, readings and interactive exercises) to course work submission and computer-based examinations. In many cases it is advocated that IT knowledge and expertise are essential transferable skills required to secure better placements in today's information society $[15,18]$. However many suggest that a significant percentage of university students find IT a source of anxiety $[25,31,49]$ even though they are constantly exposed to the overwhelming amount of IT on campus.

The argument that the digital natives [38] are better prepared to use, take advantage of IT and are better equipped to embrace innovations falls short when we examine the evidence showing that some students are forced to face high levels of anxiety on a regular basis when technology is made essential in the learning experience. This may leave students disadvantaged academically as the effect of interacting with a stressor may contribute to cognitive avoidance and off task thinking [43]. Even though new generations of students might be more familiar with the Internet, mobile technology and multi-channel information streams, it does not imply that they can carry out their learning tasks confidently and without emotional discomfort. In education, there is an implicit acceptance that the introduction of learning technologies can bring an improvement in the learning experience and make teaching more effective and efficient [14] but often the effectiveness of new tools is shadowed by the enthusiasm of the instructor pushing the innovations through. Recently, with the wide proliferation of Web 2.0 tools (which are mainly collaborative and interactive) instructors are avidly exploring the possibilities offered, often ignoring the potential negative affect this might instill in their student body. The introduction of Web 2.0 tools, such as wikis, to HE is a growing phenomenon. Due to their collaborative nature wikis offer an opportunity for students and staff to contribute and collaborate on course resources and benefit from other users expertise. Their interactivity allows users to become co-creators of course content. However due to its 
form and certain features of the wiki system interface, wikis may be a source of anxiety for users. The aim of this study is to explore anxiety towards wiki systems and its relationship with users' usability evaluation. Yet the main aim is to discover whether increased exposure to the wiki system effects users' anxiety and usability evaluation.

In the current human-computer interaction literature, much can be learned from studies on computer anxiety and Internet anxiety. Computer anxiety has been detected in student populations consistently throughout twenty years of research. [10,12,21,31,37]. In recent years, within the student population, almost 1 in 10 students were also reported to suffer from Internet anxiety [25].

Findings with regard to the increased exposure and experience on IT anxiety alleviation are mixed. Much research has highlighted that increased experience decreases computer anxiety within subjects [9], and that experience leads to liking computers [2]. Experience with computers also significantly predicts computer anxiety within individuals [16]. Experience has also been shown to correlate negatively with computer anxiety [21]. Users who report computer anxiety symptoms also use University IT services less and regular home use of computer systems was a significant predictor of computer anxiety levels within users [31]. With respects to Internet anxiety, amount of computer use per week has been shown to be negatively related to the amount of Internet anxiety individuals have [6]. On the other hand previous studies have found no such relationship between experience and anxiety reduction [39] and no relationship between use and anxiety of users [10].

Findings of experience in terms of computer and Internet anxiety allow us to gather insight into potential effects of experience in terms of anxiety reduction in an IT context. However the vagueness of the term computer anxiety leaves such findings lacking in specificity and applicability. The findings above infer the assumption that all experiences with a computer are similar. In fact over the past 20 years since computer anxiety research commenced, the uses of computers have multiplied with many different types of experiences occurring. Indeed the term computer anxiety itself betrays the complexity of computer use in society. It may not be the computer which users find anxiety inducing but the use of a certain application or interface they are attempting to interact with. The research here aims to remedy this by focusing on a specific anxiety to a specific system, the wiki. In particular wikis, due to their collaborative nature, offer an opportunity for students and staff to contribute and collaborate on course resources and benefit from other users expertise. However due to the format and certain features of the wiki system, users may hold negative emotions towards them. As wikis are not yet commonplace in HE [1] the study of any negative affective reaction that users may have and how to alleviate this is important before wikis, like the Internet and other computer uses in education, become pervasive.
A wiki is completely modifiable in its content, structure and navigational layout. All users can add, edit and amend information, structure and navigation relatively easily through use of wiki markup and/or a rich text editor. Such flexibility however may make users anxious about wiki use. The system is fluid which leaves aspects of the interaction with the system uncertain to users upon each new interaction. The addition of inappropriate content, plagiarism and unintentional deletion are all concerns which may be encountered in an educational context [1] and may lead users to question the integrity of information supplied. Used as form of class support, the fact that editors/contributors are students, inexperienced in their field of research, may leave other students (peers) uncertain over accuracy of information supplied. Concerns about the accuracy of information and neutrality of articles have been voiced in previous research into the use of wikis in university courses [13]. Social evaluation by other users may also be of concern. Students may feel anxious about changing a fellow student's information or editing the interface for fear of offending their classmates or giving students wrong information. As amendments may be attributed to users and need to if wikis are to be used as part of assessment exercises (such as collaborative writing assessments) students may feel negatively about adding and amending content in the fear of peer judgment. User attribution of edits may lead to respect and considered information addition [35] but if may also discourage users from editing the wiki [27] for fear of being judged by their peers.

Wikis also have an element of jargon and programming language associated with them, which may again lead to negative emotions. To gain full benefits from a wiki above basic functionality offered by the rich text editor, wiki markup language is needed [27]. In fact in some cases wiki markup is the sole interface used to edit a wiki. Although many feel that the simplicity of wiki markup facilitates use by wider populations $[19,27]$ it is still a language that holds specific commands and syntax which needs to be learned and is confusing to users who encounter the system for the first time [1]. Jargon and programming language learning have been factors ever present in measurements of computer anxiety and Internet anxiety [6,21,39] and may also lead to negative affect in a wiki context.

Anxiety towards wikis may have important ramifications for users' assessment of the usability of the wiki interface. Additionally, the user interface may affect the users' subsequent anxiety levels. A difficult to use interface would presumably influence the amount of anxiety experienced on first and subsequent interaction, which may influence future anxiety levels [50]. Additionally, anxiety towards certain systems may affect the evaluation of the usability of that particular IT system. Users who are more anxious about wiki use may rate the system as less usable based on their anxiety feelings towards the system. These evaluations may be 
legitimate evaluations as users find interaction with the interface uncomfortable and effort prone because of their negative affective response on interaction. The relationship between IT anxieties and usability evaluation has been relatively ignored in both research areas. The aim of usability research is to centralise the user and their desired task into the interface design process [34] and aims to ensure the user can complete their task effectively, efficiently and with satisfaction [17]. Findings focusing around concepts related to usability (such as perceived ease of use) and computer anxiety have suggested that anxiety is influential in the level at which people find an interface easy to use [41,47] and affects the quality perception of web interfaces [23]. Previous measures used in usability research have also demonstrated that affective reaction is an important criteria in the measurement of usability and user satisfaction [36,51].

The relation of experience and usability evaluation suggests that elements of usability such as perceived ease of use are positively related to experience of the system [20]. That is the more experience with the system, the more one perceives the system as being free from effort. Usability evaluation in terms of effectiveness, efficiency and frustration with the system has been seen to change as users move from novice to expert with increased use of the system $[26,33]$. However usability research tends to focus on the measurement of satisfaction over short interactions with specific systems rather than interactions with the same system over time [22]. It is important for the usability research community to develop an understanding of the effects of experience on usability and user satisfaction so a full and realistic view of the user experience can be gathered.

Before analysing the relationships between wiki usability, wiki anxiety and experience, we aim to ensure that the measures included are measuring the concepts intended effectively through the testing of internal consistency reliability and concurrent validity.

Secondly, our research aims to investigate the relationship between wiki anxiety and usability evaluation. Specifically, we hypothesise that user usability evaluation at time 1 and 2 will be significantly correlated to a user's wiki anxiety rating at both time 1 and time 2 respectively.

Thirdly, this study also aims to observe the effect of experience on wiki anxiety and usability. It is hypothesised that anxiety will change significantly with added experience of the wiki system. It is also hypothesised that usability evaluation will change after experiencing the system as users become familiar with the system. Also any change to wiki anxiety over the period of the study is hypothesised to be significantly correlated to a change in wiki usability evaluation.

\section{METHOD}

\subsection{Participants}

Participants were 92 Second year undergraduate psychology students at the University of Edinburgh who used a wiki (Atlassian Confluence- known within the course as Psykowiki) as part of a course requirement for the Psychology 2 Methodology and Statistics practical course. The wiki was aimed at helping collaboration within and between students groups and staff for the end of term group projects. Of these 92 participants, 75 $(81.5 \%)$ were female and $17(18.5 \%)$ were male. The age range of the sample was $19-39$ with a mean age of 20.72 years.

\subsection{Questionnaires and Procedure}

The online survey was initially administered 2 weeks after students were first introduced to Psykowiki (Time 1). A wiki page design task was given to the students to complete within these two weeks which meant students had the opportunity to experience the wiki before assessing its usability and their anxiety towards it. The same online survey was then administered after the submission of group projects, 12 weeks into the term, 10 weeks after the administration of the initial online survey (Time 2). The surveys were completed as part of a course assignment. Of the initial 125 participants at time 1,96 completed all questionnaires at both time 1 and 2. Of the 96, 4 were excluded from the sample as they had answered 0 to the average amount of hours they had used the wiki per week at time 1. All users therefore had some experience with the wiki interface when taking part in the study.

Inventories included in the online survey were a 14 item version of the Wiki Anxiety Inventory [11], the Wiki Usability Inventory [11] and a short form (6 item) version of the state facet of the State-Trait Anxiety Inventory (STAI) [30].

The Wiki Anxiety Inventory (WAI) is a self report measure based on previous IT anxiety inventories and research on computer and Internet anxiety [21,25,45]. The measure consisted of 7 positive items (such as "I hope that soon everyone will be using wikis") and 7 negative items (such as "I hesitate to use a wiki for fear of making mistakes that I cannot correct"). The items included made reference to aspects such as confidence when using the wikispace, interaction anxiety, learning anxiety, judgment by peers and information concerns. Positive items were reverse scored so that a low score would suggest a low anxiety level towards wikis. All negative and positive items scores were then summed to give a total wiki anxiety score. The possible scale scores range from a minimum of 14 to a maximum of 70 .

The Wiki Usability Inventory (WUI) includes 31 items (16 positive and 15 negative) referring to aspects 
influential in the user experience of the wikispace. This measure was influenced by previous research into self report usability evaluation and satisfaction [8,36,51] and measured elements of user interaction such as structure and navigation, clarity, emotional reaction to interface as well as the rated effectiveness of the interface in allowing the user to achieve their desired task. With this measure, negative items were negatively marked to allow a large usability score to reflect a positive evaluation of the interface. To ensure concurrent validity a question measuring user experience with the wikispace was also included. This asked users to rate on a scale of 1-10 their experience with the wiki site. The possible scale scores for the WUI range from a minimum of 31 to a maximum of 155 .

The STAI-short was included to measure concurrent validity of the WAI. As the other self report questionnaires within the survey were of considerable length, it was decided that this short form would minimise the chances of question fatigue compromising the quality of data gathered. The STAI short is noted as sensitive to self report state anxiety differences and highly reliable $(\alpha=.82)$ [30]. It includes 3 positive items (such as "I feel content") and 3 negative items (such as "I am worried") originally included in the state dimension of the STAI anxiety measure [44]. To calculate the state anxiety score positive items were reverse scored and added with negative items scores to gain a total state anxiety score. Positive items were negatively marked to allow for a large score to reflect a high amount of state anxiety. The possible scale scores range from a minimum of 6 to a maximum of 30 .

All inventories used a 5-point Likert scale ranging from Strongly Disagree to Strongly Agree to measure the amount each participant agreed that each item applied to them.

Demographic information such as gender, age and the average amount each user used the wiki per week (Wiki Experience) were gained. Participants were asked "On average how many hours do you use the wiki per week?" at time 1 and at time 2 so as to observe any change in experience over the experiment.

\section{RESULTS}

\subsection{Data Consideration}

Before analysing the data, Kolmogorov-Smirnov (K-S) tests were run on each of the variables within the study to assess whether violations in normality occurred in the data gathered. The results of these tests (K-S Sig) are displayed in Tables 1, 2 and 3. Non-parametric analyses were run in parallel to parametric analyses when testing hypotheses involving variables which violated normality assumptions. There was however no differences in the levels of significance achieved between both parametric and non-parametric analyses. It was therefore decided to present the parametric analysis so as to include quantitative interval based analysis rather than analysis based on ranked data.

\subsection{Questionnaire Reliability and Validity}

The internal consistency reliability of the scales was good in both conditions with high Cronbach alpha values recorded (Time 1- STAI: $\alpha=.90$; WAI: $\alpha=.83$; WUI: $\alpha=.93$; Time 2- STAI: $\alpha=.89$; WAI: $\alpha=.82$; WUI: $\alpha=.95$ ). Concurrent validity for both the WAI and WUI in both conditions was also satisfactory. A significant positive correlation was found between WAI and STAI measures [Time 1: $\mathrm{r}(90)=.68, \mathrm{p}<0.001$; Time $2: \mathrm{r}(90)=$ $.40, \mathrm{p}<0.001]$. The WUI correlated highly with the conceptually related measure of participant's experience rating [Time 1: $\mathrm{r}(90)=.66, \mathrm{p}<0.001$; Time $2: \mathrm{r}(90)=$ $.0 .67, \mathrm{p}<0.001]$. The means of all variables included in the validity analysis are displayed in Table 1.

Table 1: Mean Wiki Anxiety, Wiki Usability, State Anxiety and Experience Rating at Time 1 and Time 2

\begin{tabular}{|l|c|c|c|c|}
\hline \multicolumn{1}{|c|}{ Measure } & N & Mean & S.D. & K-S Sig \\
\hline Wiki Anxiety Score (Time 1) & 92 & 38.17 & 8.35 & $>0.05$ \\
\cline { 2 - 5 } Wiki Anxiety Score (Time 2) & 92 & 37.84 & 8.34 & $>0.05$ \\
\hline Usability Score (Time 1) & 92 & 97.01 & 17.49 & $>0.05$ \\
\cline { 2 - 5 } Usability Score (Time 2) & 92 & 95.67 & 21.66 & $>0.05$ \\
\hline $\begin{array}{l}\text { State Anxiety Score (Time 1) } \\
\text { State Anxiety Score (Time 2) }\end{array}$ & 92 & 12.79 & 4.67 & $<0.05$ \\
\cline { 2 - 5 } Experience Rating (Time 1) & 92 & 12.28 & 4.58 & $<0.001$ \\
\cline { 2 - 5 } Experience Rating (Time 2) & 92 & 5.12 & 1.77 & $<0.001$ \\
\hline
\end{tabular}

\subsection{Relationship between Wiki Anxiety and Usability Evaluation}

A bivariate correlation analysis revealed that at both time 1 and time 2 a significant negative correlation exists between wiki anxiety and wiki usability (Time $1: \mathrm{r}(90)=$ $-.69, \mathrm{p}<0.001$; Time 2: $\mathrm{r}(90)=-.83, \mathrm{p}<0.001)$. Users who hold high wiki anxiety also rate the wiki as less usable whereas those with less anxiety rate the wiki as more satisfactory to use. The hypothesis that usability rating at both time 1 and 2 is significantly correlated to wiki anxiety at time 1 and 2 was supported.

\subsection{Effects of experience on Wiki Anxiety and Usability Evaluation}

Before directly testing our hypotheses of the effects of experience on usability and wiki anxiety, a paired sample t-test was run on the reported amount of hours per week using the system to observe if there was any significant difference between average wiki use per week at time 1 and time 2. The mean average wiki use per week for time 1 and 2 is presented in Table 2 below. 
Table 2- Mean wiki use per week at week 2 (Time 1) and week 12 (Time 2)

\begin{tabular}{|c|c|c|c|c|}
\hline Measure & $\mathbf{N}$ & Mean & S.D. & K-S Sig. \\
\hline $\begin{array}{llll}\text { Wiki Experience (Hrs } & \text { Per } \\
\text { Week) } & \text { (Time 1) }\end{array}$ & 92 & 2.10 & 1.01 & $<0.001$ \\
\hline $\begin{array}{llll}\text { Wiki } & \text { Experience } & \text { (Hrs } & \text { Per } \\
\text { Week) } & \text { (Time 2) }\end{array}$ & 92 & 2.29 & 1.28 & $<0.001$ \\
\hline
\end{tabular}

On observation of the mean, average wiki use per week increased slightly over the 10 week period of the study. The results of the t-test analysis suggest that wiki use per week did not significantly change over the course of the experiment [t $(91)=-1.41, p>0.05]$. Average use per week did not change significantly over the 10 -week period between time 1 and 2 . Although there may be no change in hours using the wiki per week, users' overall experience with the system should still have increased over this 10 -week period at an average rate of approximately 2 hrs per week. Conclusions can therefore still be made about the effect of increased experience of the system and the dependent variables measured.

Two paired sample t-tests were run to test the hypotheses that users wiki anxiety and wiki usability rating would significantly differ between time 1 and time 2. The means in Table 1 above imply that there is little difference between condition 1 and 2 in either wiki anxiety or wiki usability rating. No significant difference was found between the anxiety experienced at time 1 and the anxiety experienced at time $2[\mathrm{t}(91)=0.47, \mathrm{p}>0.05]$. Our hypothesis that wiki anxiety would significantly differ from time 1 and time 2 was not supported. Our hypothesis that usability evaluation would differ from time 1 to time 2 was also not supported $[\mathrm{t}(91)=0.72$, $\mathrm{p}>0.05]$. There was no significant difference between usability evaluation at time 1 and time 2 .

Further analysis was conducted to observe the relationship between changes in reported hours per week using the wiki (wiki experience), wiki anxiety and wiki usability rating over time 1 and time 2 .

Table 3: Mean change in Wiki Anxiety, Wiki Usability and Wiki Experience across experiment

\begin{tabular}{|l|c|c|c|c|}
\hline \multicolumn{1}{|c|}{ Measure } & N & Mean & S.D. & K-S Sig \\
\hline Wiki Anxiety Change & 92 & -0.33 & 6.84 & $>0.05$ \\
\hline Wiki Usability Change & 92 & -1.33 & 17.84 & $>0.05$ \\
\hline $\begin{array}{l}\text { Wiki Experience Change (Hrs } \\
\text { Per Week) }\end{array}$ & 92 & 0.19 & 1.33 & $<0.01$ \\
\hline
\end{tabular}

From the means presented in Table 3 it is clear that there is very little change on all variables between time 1 and time 2, further supporting the insignificant findings on the effects of experience on wiki anxiety and wiki usability reported above. A bivariate correlation analysis showed that changes in wiki anxiety were significantly negatively correlated with changes in usability rating $[\mathrm{r}$ $(90)=-.59, \mathrm{p}<0.001]$. There was therefore a negative relationship between change in usability and anxiety towards the wiki system. There was no significant relationship between change in wiki usability rating, wiki anxiety and change in average hours per week spent on the wiki [Wiki anxiety change: $\mathrm{r}(90)=-.15, \mathrm{p}>0.05 ;$ Wiki usability change: $\mathrm{r}(90)=.06, \mathrm{p}>0.05]$

In terms of the effects of experience, our analysis has found that wiki anxiety and wiki usability rating do not significantly change with more exposure to the system. However any change in usability rating is significantly negatively related to a change in wiki anxiety. There is no significant relationship between change in experience and both wiki anxiety and wiki usability rating.

\section{DISCUSSION}

In summary the Wiki Anxiety Inventory (WAI) and the Wiki Usability Inventory (WUI) measures showed high internal consistency reliability and concurrent validity through their high Cronbach alpha coefficients and their correlation with other self report measures of anxiety and user satisfaction. Wiki anxiety was also found to significantly negatively correlate with wiki usability. In terms of the effects of experience on anxiety and usability, findings from this study suggest that anxiety did not significantly change after more experience with the wiki system. Usability rating also did not significantly change over the period of the study. Users' changes in wiki anxiety were also negatively related to their changes in wiki usability.

Before discussing our findings in reference to the relationship between anxiety, usability and experience some notes of caution must be made about the interpretation of these results. Although our questionnaires have demonstrated high internal consistency and good concurrent validity, other criteria for reliability and validity need to be demonstrated to be sure of the psychometric quality of these measures. For instance, further research including measures predicted to correlate with wiki anxiety such as social anxiety and experiment based manipulations of wiki anxiety and usability would allow for further demonstration of the validity of the wiki anxiety measure used. Additionally, although the non-significant findings are discussed below, it must be noted that these findings are specific to this sample. Furthermore the sample size limits the power of our statistical analysis to the discovery of medium effect sizes within the data [24]. A larger, more varied sample of wiki users should be tested in future research to identify whether these findings can be generalized and applied more widely.

Although our analysis suggests a relationship between wiki usability and wiki anxiety exists, because of the nature of the analysis it is unclear how these variables influences each other. It may be that a poor user 
experience leads to increased anxiety about further interactions with the wiki system. Research in computer anxiety highlights the importance of good first experiences with computers in general as a precursor to lower computer anxiety later [46] and that a user friendly experience with a computer is an important factor in alleviation [52]. A bad experience with a wiki may be the catalyst for anxious feeling towards that type of system.

Our finding may also be interpreted as user's predisposition for anxiety (trait anxiety) affecting their usability evaluation. All users in this study were evaluating the same system and individual differences are likely to lead to differing subjective experiences whilst using the wiki system. Users' propensity for anxious feeling may lead to more negative evaluations of the interfaces quality and affect levels of satisfaction with the wiki interface. A relationship between trait anxiety and computer anxiety has been found previously [3]. Further research is needed on how wiki anxiety relates to a users' predisposition towards anxiety and how that may affect usability rating. What can be concluded from this research is that a strong negative relationship between wiki anxiety and usability evaluation exists. This is further emphasised by the negative relationship between changes in anxiety and usability. Further investigation is needed to determine the cause and effect of this relationship.

Our findings also suggest that expecting students to become comfortable with prolonged exposure may be an ineffective way to relieving any anxiety users may have about wiki systems. This is contrary to what is inferred from previous research in the area of computer anxiety and experience [9]. In fact the effect of experience may be influenced more by quality of experience rather than amount gathered. Research has demonstrated that initial exposure to computers should be in a relaxed and fun atmosphere to lead users to use computers more and in turn reduce negative affect towards computers [2]. Also experiencing high impact positive interactions may allow users who are anxious about computers to become less so [46]. Experiences that heighten users' confidence when using the system should therefore be sought. Self efficacy $^{1}$ 's negative relationship with computer anxiety is well documented [4,7,12]. Other IT anxiety research suggests that anxiety may be due to self efficacy rather than lack of experience [32]. Interactions that would increase users' self-efficacy could reduce anxiety levels in wiki anxious users. In this case, due to the design of the experiment, the authors were unable to control or monitor the quality or type of experiences each user had when using the wiki. Controlled studies where users are asked to conduct specific tasks in a controlled wiki interaction would allow for clearer conclusions on the effect of amount and quality of experience each user gains on wiki anxiety.

\footnotetext{
${ }^{1}$ One's judgment of their ability to conduct a specific task [29].
}

As mentioned, quality of experience may be more important than the amount of experience. Usability is of critical importance in the success and quality of first experience. Faulty or badly designed software is doubtless influential on anxiety levels [40] and self efficacy judgments made after first experience because of the systems unpredictability and difficulty to use. The wiki user interface has been noted as a problem for wiki users in previous research [48]. The less usable a system is the more likely that a user's first experience will be unsuccessful and their confidence in their ability to use that system will be low. It is as yet unclear whether a more usable system would reduce the onset of anxiety. Our research shows that users rated the usability of the wiki system in question (which included items related to the viewing and editing interface) as moderate. Using an interface with such a rating may have instilled anxiety into the users after their first experience that carried to their further experiences with the system. Research comparing interfaces that differ in terms of usability needs to be conducted in this area to ascertain whether such a hypothesis is justified.

Further research into aspects of the interaction which may contribute to negative emotions is also needed to ascertain which elements of the experience users may find anxiety inducing. Wikis are multi-user systems where knowledge is built through group collaboration. Factors such as judgment by peers in contribution, group dynamics and reputation and fluidity of information may lead to emotional arousal in wiki use and affect satisfaction in interaction. Future research should be conducted to monitor the effects these fundamental aspects of wiki systems have on the concept of wiki anxiety and users' satisfaction in interaction. Such research could give a valuable and more developed insight into the wiki user experience. This insight may be crucial for successful implementation of wiki systems within higher education and reducing negative user emotion.

The research presented also suggests no significant changes in satisfaction over time. This finding allows a tentative insight into the effect of time on satisfaction, something rarely tested in usability research [22]. It suggests that even with increased exposure to the wiki system over the ten weeks of the experiment, users' satisfaction tended not to change significantly. Users' assessment of satisfaction in the initial setting may thus be robust and inflexible to time spent using the interface. This result may be explained by our findings in terms of anxiety over the experiment. Because anxiety did not significantly change, it is unlikely that usability evaluation will significantly change as users still feel anxious about using the interface further. Alternatively an interaction with a poor system throughout the time of interaction may not have lead to much of a change in anxiety levels as the system has not changed in usability terms. The interpretation depends on whether anxiety towards the wiki is trait related or whether it is system 
initiated. If it is the latter we would expect a consistently poor system which does not change within that time to instill similar levels of negative affect in its users. However if it is trait related then wiki anxiety may be more deep-seated and be affecting usability evaluation due to the individual difference of propensity towards anxiety. Again further research into the nature of the relationship between anxiety and usability is needed before conclusions can be made on such claims.

Further research is also needed to determine the effects of wiki anxiety on other usability factors such as effectiveness and efficiency. As shown in computer anxiety research, wiki anxiety may have negative cognitive and performance effects such as off task thinking [42] and task completion accuracy [5]. Users who are computer anxious were also shown to be slower at completing computer based data entry tasks [28]. It is unclear at present how such an anxiety may affect users in a wiki context. Research focusing on performance measures when using wikis is needed to develop a clear view of the performance effects wiki anxiety may have on users.

In conclusion, the above research demonstrates that a negative relationship exists between how anxious users are about wikis and how they rate their satisfaction with the wiki system. More experience with the interface did not tend to affect wiki anxiety nor did it significantly affect how users rated the system in terms of usability. It therefore may be futile to expect that users will reduce in anxiety solely by gaining more experience with the systems introduced. It is maybe ineffective to expect students' anxiety to dissipate without a careful and considered approach to training and support throughout the students' use of such systems, although more research is needed to be able to generalize such a conclusion. More research is needed to clarify and determine the causes of wiki anxiety, especially in terms of the role of usability of the system in first experience quality and anxiety onset as well as the effects of quality of experience on wiki anxiety after first experience. Further investigation on the impact of wiki anxiety on user performance is also needed. What this research tentatively highlights is that a considered and cautious approach is needed to the introduction and use of wikis in University. Such technologies allow users to collaborate and share resources and information which is of great benefit to staff and students. That said a focus on the human factors and emotional reactions in the introduction and continued use of such systems must become a priority if wikis are to become a significant pedagogical tool in modern higher education.

\section{ACKNOWLEDGMENTS}

This work was partially funded by the Engineering and Physical Sciences Research Council (EPSRC). The authors would like to thank Fergus McInnes, Gareth Peevers for their comments and suggestions on previous drafts of this manuscript. The authors would also like to thank the anonymous reviewers for their comments and suggestions which have helped to improve this manuscript.

\section{REFERENCES}

1. Augar, N., Raitman, R., and Zhou, W. Teaching and learning online with wikis. Beyond the comfort zone: Proceedings of the 21st ASCILITE Conference, (2004), 95-104.

2. Beckers, J.J. and Schmidt, H.G. Computer experience and computer anxiety. Computers in Human Behavior 19, (2003), 785-797.

3. Beckers, J.J., Wicherts, J.M., and Smith, H.G. Computer Anxiety: "Trait" or "State". Computers in Human Behavior 23, (2007), 2851-2862.

4. Brosnan, M.J. Technophobia; The psychological impact of information technology. Routledge, London, UK, 1998.

5. Brosnan, M.J. The impact of computer anxiety and self efficacy upon performance. Journal of Computer Assisted Learning 14, (1998), 223-234.

6. Chou, C. Incidences and correlates of Internet anxiety among high school teachers in Taiwan. Computers in Human Behavior 19, (2003), 731-749.

7. Chou, H.W. Effects of training method and computer anxiety on learning performance and self-efficacy. Computers in Human Behavior 17, (2001), 51-69.

8. Chou, J.R. and Hsiao, S.W. A usability study on humancomputer interface for middle-aged learners. Computers in Human Behavior 23, (2007), 2040-2063.

9. Chua, S.L., Chen, D.T., and Wong, A.F. Computer anxiety and its correlates: a meta-analysis. Computers in Human Behavior 15, (1999), 609-623.

10. Compeau, D.R., Higgins, C.A., and Huff, S. Social Cognitive Theory and individual reactions to computing technology: A longitudinal study. MIS Quarterly 23, 2 (1999), 145-158.

11. Cowan, B., Vigentini, L., and Jack, M.A. Exploring the relationship between anxiety and usability evaluation- An online study of Internet and wiki anxiety. Proceedings of IADIS International Conference; Interfaces and Human Computer Interaction 2008, (2008), 69-76.

12. Durndell, A. and Hagg, Z. Computer self efficacy, computer anxiety, attitudes towards the Internet and reported experience with the Internet, by gender, in an Eastern European sample. Computers in Human Behavior $18,(2002), 521-535$.

13. Ebner, M., Zechner, J., and Holzinger, A. Why is Wikipedia so successful? Experiences in establishing the principles in Higher Education. (2006), 527-535.

14. Eggers, W. Government 2.0: Using Technology to Improve Education, Cut Red Tape, Reduce Gridlock, and Enhance Democracy. New York, 2005.

15. Eisenberg, M. Information Literacy: Essential Skills for the Information Age. Journal of Library \& Information Technology 28, 2 (2008), 39-47.

16. Farina, F., Arce, R., Sobral, J., and Carames, R. Predictors of anxiety towards computers. Computers in Human Behavior 7, (1991), 263-267. 
17. Faulkner, X. Usability Engineering. Palgrave, Hampshire, UK, 2000.

18. Garnham, N. 'Information Society' as theory or ideology: A critical perspective in technology, education and employment in the information age. Information, Communication \& Society 3, 2 (2000), 139-152.

19. Goodwin-Jones, R. Emerging Technologies- Blogs and Wikis: Environments for On-line Collaboration. Language, Learning and Technology 7, 2 (2003), 12-16.

20. Hackbarth, G., Grover, V., and Mun, Y.Y. Computer playfulness and anxiety: positive and negative mediators of the system experience effect on perceived ease of use. Information and Management 40, (2003), 221-232.

21. Heinssen, R.K., Glass, C.R., and Knight, L.A. Assessing Computer Anxiety: Development and validation of the Computer Anxiety Rating Scale. Computers in Human Behavior 3, (1987), 49-59.

22. Hornbaek, K. Current practice in measuring usability: Challenges to usability studies and research. International Journal of Human-Computer Studies 64, 2 (2006), 79-102.

23. Hwang, Y. and Kim, D.J. Customer self-service systems: The effects of perceived Web quality with service contents on enjoyment, anxiety, and e-trust. Decision Support Systems 43, (2007), 746-760.

24. J. Cohen. A power primer. Psychological Bulletin 112, 1 (1992), 155-159.

25. Joiner, R., Brosnan, M., Duffield, J., Gavin, J., and Maras, P. The relationship between Internet identification, Internet anxiety and Internet use. Computers in Human Behavior 23, (2007), 1408-1420.

26. Kjeldskov, J., Skov, M., and Stage, J. Does time heal? A longitudinal study of usability. Proceedings of the19th Conference of the Computer-Human Interface Special Interest Group (CHISIG) of Australia on ComputerHuman Interaction, (2005), 1-10.

27. Mader, S. Wikipatterns; a practical guide to improving productivity and collaboration in your organization. Wiley Publishing Inc., Indianapolis, 2008.

28. Mahar, D., Henderson, R., and Deane, F. The effects of computer anxiety, state anxiety, and computer experience on users' performance of computer based tasks. Personality and Individual Differences 22, 5 (1997), 683692.

29. Marakas, G., Mun, Y., and Johnson, R. The multilevel and multifaceted character of computer self efficacy: toward clarification of the construct and an integrative framework for research. Information Systems Research 9, 2 (1998), 126-163.

30. Marteau, T.M. and Bekker, H. The development of a sixitem short form of the state scale of the Spielberger StateTrait Anxiety Inventory (STAI). British Journal of Clinical Psychology 31, (1992), 301-306.

31. Mcilroy, D., Sadler, C., and Boojawon, N. Computer phobia and computer self efficacy: their association with undergraduates' use of university computer facilities. Computers in Human Behavior 23, (2007), 1285-1299.

32. McInerney, V., McInerney, D.M., and Sinclair, K.E. Student teachers, computer anxiety and computer experience. Journal of Educational Computing Research 11, 1 (1994), 27 - 50.
33. Mendoza, V. and Novick, D. Usability over time. Proceedings of SIGDOC 2005, (2005), 151-158.

34. Nielsen, J. Usability Engineering. Academic Press, London, UK, 1993.

35. O'Neill, M.E. Automated use of a Wiki for collaborative lecture notes. SIGCSE Bulletin 37, 1 (2005), 267-271.

36. Peevers, G., Douglas, G., and Jack, M.A. A usability comparison of three alternative message formats for an SMS banking service. International Journal of HumanComputer Studies 66, (2008), 113-123.

37. Popovich, P.M., Gullekson, N., Morris, S., and Morse, B. Comparing attitudes towards computer usage by undergraduates from 1986 to 2005. Computers in Human Behavior 24, (2008), 986-992.

38. Prensky, M. Digital Natives, Digital Immigrants. In On the Horizon. NCB University Press, 2001.

39. Rosen, L.D., Sears, D.C., and Weil, M.M. Computerphobia. Behavior Research Methods, Instruments, \& Computers 19, 2 (1987), 167-179.

40. Rosen, L.D. and Weil, M.M. What We Have Learned From a Decade of Research (1983-1993) on "The Psychological Impact of Technology". Computers and Society, (1994), 3-9.

41. Saade, R.G. and Kira, D. Mediating the impact of technology usage on perceived ease of use by anxiety. Computers \& Education 49, (2007), 1189-1204.

42. Smith, B. and Caputi, P. Cognitive interference in computer anxiety. Behaviour \& Information Technology 20, 4 (2001), 265-273.

43. Smith, B. and Caputi, P. Cognitive interference model of computer anxiety: Implications for computer-based assessment. Computers in Human Behavior 23, (2007), 1481-1498.

44. Spielberger, C.D. Manual for the State-Trait Anxiety Inventory (Form Y). Consulting Psychologists Press, Palo Alto, CA, 1983.

45. Thatcher, J.B., Loughry, M.L., Lim, J., and McKnight, H. Internet anxiety: An empirical study of the effects of personality, beliefs, and social support. Information \& Management 44, (2007), 353-363.

46. Todman, J. and Drysdale, E. Effects of qualitative differences in initial and subsequent computer experience on computer anxiety. Computers in Human Behavior 20, (2004), 581-590.

47. Venkatesh, V. Determinants of perceived ease of use: Integrating control, instrinsic motivation, and emotion into the Technology Acceptance Model. Information Systems Research 11, 4 (2000), 342-365.

48. Wagner, C. Wiki: A technology for conversational knowledge management and group collaboration. Communications of the Association for Information Systems 13, (2004), 265-289.

49. Weil, M.M. and Rosen, L.D. The psychological impact of technology from a global perspective: A study of technological sophistication and technophobia in University students from twenty-three countries. Computers in Human Behavior 11, 1 (1995), 95-133.

50. Weil, M.M., Rosen, L.D., and Wugalter, S.E. The Etiology of Computerphobia. Computers in Human Behavior 6, (1990), 361-379. 
Exploring the Effects of Experience on Wiki Anxiety and Wiki Usability: An Online Study

51. Weir, C.S., Anderson, J.N., and Jack, M.A. On the role of metaphor and language in design of third party payments in eBanking: Usability and quality. International Journal of Human-Computer Studies 64, (2006), 770-784.
52. Wilson, B. Redressing the anxiety imbalance: computerphobia and educators. Behaviour and Information Technology 18, 6 (1999), 445-453. 